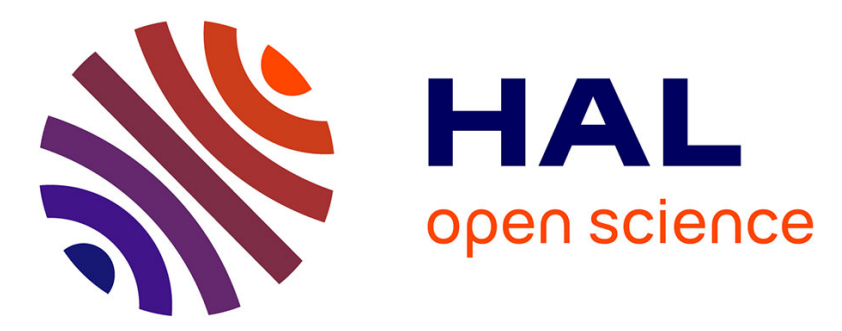

\title{
On the Interaction between Heterogeneity and Decay in Two-way Flow Models
}

\author{
Pascal Billand, Christophe Bravard, Sudipta Sarangi
}

\section{To cite this version:}

Pascal Billand, Christophe Bravard, Sudipta Sarangi. On the Interaction between Heterogeneity and Decay in Two-way Flow Models. 2011. halshs-00574258

\section{HAL Id: halshs-00574258 \\ https://shs.hal.science/halshs-00574258}

Submitted on 7 Mar 2011

HAL is a multi-disciplinary open access archive for the deposit and dissemination of scientific research documents, whether they are published or not. The documents may come from teaching and research institutions in France or abroad, or from public or private research centers.
L'archive ouverte pluridisciplinaire HAL, est destinée au dépôt et à la diffusion de documents scientifiques de niveau recherche, publiés ou non, émanant des établissements d'enseignement et de recherche français ou étrangers, des laboratoires publics ou privés. 
On the Interaction between Heterogeneity and Decay in Two-way Flow Models

Pascal Billand, Christophe Bravard, Sudipta Sarangi

Mars 2011 


\section{GATE Groupe d'Analyse et de Théorie Économique Lyon-St Étienne}

93, chemin des Mouilles 69130 Ecully - France

Tel. +33 (0)4 72866060

Fax $+33(0) 472866090$

6, rue Basse des Rives 42023 Saint-Etienne cedex 02 - France

Tel. +33 (0)4 77421960

Fax. $+33(0) 477421950$

Messagerie électronique / Email : gate@gate.cnrs.fr

Téléchargement / Download : http://www.gate.cnrs.fr - Publications / Working Papers 


\title{
On the Interaction between Heterogeneity and Decay in Two-way Flow Models*
}

\author{
PASCAL BILLAND $^{a}$, CHRISTOPHE BRAVARD $^{a}$, SUDIPTA SARANGI ${ }^{b}$
}

${ }^{a}$ Université de Lyon, Lyon, F-69003, France ; Université Jean Monnet, Saint-Etienne, F-42000, France ; CNRS, GATE Lyon St Etienne, Saint-Etienne, F-42000, France. email: pascal.billand@univ-st-etienne.fr, christophe.bravard@univ-st-etienne.fr

${ }^{b}$ DIW Berlin and Department of Economics, Louisiana State University.

email: sarangi@lsu.edu

* We thank Hans Haller, Matt Jackson, Brian Rogers and Santanu Roy for useful suggestions. We also thank seminar participants at Virginia Tech, University of Virginia, Southern Methodist University, University of Wisconsin-Milwaukee and LGS 5 for helpful comments.

Sudipta Sarangi acknowledges the support of NSF grant HSD-0527315 and the hospitality of Gate Lyon-Saint-Etienne and Jean Monnet University.

\begin{abstract}
In this paper we examine the role played by heterogeneity in the popular "connections model" of Jackson and Wolinsky (1996). We prove that under heterogeneity in values or decay involving only two degrees of freedom, all networks can supported as Nash. Moreover, we show that Nash networks may not always exist. In the absence of decay, neither result can be found in a model with value heterogeneity. Finally, we show that on reducing heterogeneity, both the earlier "anything goes" result and the non-existence problem disappear.
\end{abstract}

JEL Classification: C72, D85.

Key Words: connections model, decay, two-way flow models 


\section{Introduction}

The connections model of Jackson and Wolinsky (1996) is one of the most popular models in network theory. In this model an agent acquires information from all the agents she is directly or indirectly linked to and pays a fixed cost for all her direct links. However, the model allows for partial transitivity of resources that an agent obtains. First, the information she obtains from her direct links is weighted by a decay parameter. Indirect links yield still lower benefits in the following manner from those who are two links away the information is weighted by the square of the decay parameter, from those three links away by its cube, and so on. Since information loses value as it travels along a sequence of links, the decay parameter captures the idea that "it is better to have the facts straight from the horse's mouth." Bala and Goyal (2000) studied Nash as well as strict Nash networks for the homogeneous (values, costs of link formation and decay) parameters version of this model. ${ }^{1}$

In this paper we introduce heterogeneity in the model by first allowing for value heterogeneity, keeping the link cost as well as the decay parameter same for all links. A second version of the model allows for heterogeneity in the decay parameter while keeping values and costs constant across all links. Heterogeneity of players and links arise quite naturally in information networks. For example, some individuals are better informed which makes them more valuable as contacts. This can be understood as heterogeneity of players in the model. Similarly the extent of the communication between pairs of individuals can vary, since it is often easier to communicate with some individuals than with some others. Thus the amount of

\footnotetext{
${ }^{1}$ A decay model for Nash networks has recently been analyzed by Hojman and Szeidl (2008). In this model, costs of links are homogeneous and linear, while the benefit function is quite general. The authors assume that the benefit function is strictly increasing and concave. Moreover, the benefits from links further away go to zero at some threshold level of distance. The authors find that Nash networks tend to have a core-periphery structure. Due to the nature of their payoff function however, we are unable to compare our results with them. Instead, we restrict attention to the papers mentioned in the introduction which utilize the linear payoff specification of Bala and Goyal (2000).
} 
information acquired will depend on the $\operatorname{link}(\mathrm{s})$ through which it travels, allowing us to model this as link heterogeneity. The paper studies the existence and characterization of (strict) Nash networks for these two types of heterogeneity.

Heterogeneity in Nash networks has been studied by Galeotti, Goyal and Kamphorst (2006) and Haller, Kamphorst and Sarangi (2007). Both these models investigate the consequences of heterogeneity for the full transitivity (or no decay) model of Nash networks with homogeneous parameters introduced to the literature by Bala and Goyal. In the Bala and Goyal model with no decay, each agent pays only for her direct links and is able to acquire the full value of information of all agents she is directly or indirectly linked to. Haller, Kamphorst and Sarangi introduce heterogeneity in the value and cost parameters to study the existence of Nash networks. Galeotti, Goyal and Kamphorst also introduce heterogeneity in values and costs but focus on the characterization of strict Nash networks.

The contribution of this paper to the literature can be summarized in the following manner. When decay and costs are homogeneous while the link between player $i$ and player $j$ provides value $V_{i, j}$ to player $i$ we find that:

1. Nash networks may not always exist. This is in sharp contrast to Haller, Kamphorst and Sarangi who find that in models of full transitivity, value heterogeneity does not lead to non-existence. Non-existence occurs only with cost heterogeneity. However, if we reduce heterogeneity by allowing $V_{i, j}=$ $V_{i}$, then it turns out that Nash networks always exist. This result is also interesting for another reason. As a corollary it tells us that Nash networks always exist in the homogeneous parameters model of Bala and Goyal (2000) - an issue that these authors had left unresolved in their paper.

2. All networks can be supported as strict Nash networks. This result echoes the full transitivity model of Galeotti, Goyal and Kamphorst with cost heterogeneity which shows that strict Nash networks must be minimal as well as 
its converse. ${ }^{2}$ We know that under full transitivity (regardless of parameters values) with costly link formation, the set of potential strict Nash networks ${ }^{3}$ consists of networks without any links that do not provide access to new players. With cost heterogeneity, Galeotti, Goyal and Kamphorst show that the set of strict Nash networks coincides with the set of potential strict Nash networks, i.e., the equilibrium set is maximal. We find that the same is true in our model. Hence with partial transitivity value heterogeneity functions like cost heterogeneity with full transitivity.

3. In general, we find that when we reduce the magnitude of heterogeneity or set $V_{i, j}=V_{i}$, the "anything goes" results disappear. Moreover, in this case we find that strict Nash networks must contain only one component. However, unlike Galeotti, Goyal and Kamphorst where all players must belong to some component, isolated players can exist in our model.

The model with heterogeneous decay and homogeneous cost and values yields similar results. Therefore these results are presented very briefly in the paper.

The rest of the paper is organized as follows. Section 2 introduces the model. Section 3 presents the results on Nash networks in models with decay and heterogeneity.

\section{Model Setup}

In this section we define the formal elements of the strategic form network formation game. Let $N=\{1, \ldots, n\}, n \geq 3$, denote the set of players with generic elements $i, j, k$.

Strategies. For player $i$ a pure strategy is a vector $\boldsymbol{g}_{i}=\left(g_{i, 1}, \ldots, g_{i, i-1}, g_{i, i+1}\right.$, $\left.\ldots, g_{i, n}\right) \in\{0,1\}^{n-1}$. Since our aim is to model network formation, $g_{i, j}=1$ implies

\footnotetext{
${ }^{2}$ Minimal networks consist of components without any links that do not provide access to new players.

${ }^{3}$ That is the set of networks such that there exist parameters which allow these networks to be Nash.
} 
that there exists a direct link between $i$ and $j$ initiated by player $i$; this link is denoted by $i j$. If $g_{i, j}=0$, then $i$ does not initiate a link with $j$. Regardless of what player $i$ does, player $j$ can always choose to initiate a link with $i$ or set $g_{j, i}=0$. Here we focus only on pure strategies. The set of all pure strategies of player $i$ is denoted by $\mathcal{G}_{i}$ and consists of $2^{n-1}$ elements. The joint strategy space is given by $\mathcal{G}=\mathcal{G}_{1} \times \cdots \times \mathcal{G}_{n}$. Note that there is a one-to-one correspondence between $\mathcal{G}$ and the set of all simple directed graphs (that is graphs with no loops and there is at most one directed link from a player $i$ to a player $j$ ) or networks with vertex set $N$. Namely, to a strategy profile $\boldsymbol{g}=\left(\boldsymbol{g}_{1}, \ldots, \boldsymbol{g}_{n}\right) \in \mathcal{G}$ corresponds the graph $(N, E(\boldsymbol{g}))$ where $E(\boldsymbol{g})$ is the set of links which satisfies $i j \in E(\boldsymbol{g})$ iff $g_{i, j}=1$. In the sequel, we identify a joint strategy $\boldsymbol{g}$ by its corresponding graph and use the terminology directed graph or network $\boldsymbol{g}$ for it.

Network definitions. The closure of $\boldsymbol{g}$, denoted by $\overline{\boldsymbol{g}} \in \mathcal{G}$, is defined by $\bar{g}_{i, j}=\max \left\{g_{i, j}, g_{j, i}\right\}$ for $i \neq j$. The closure of $\boldsymbol{g}$ is thus the undirected counter-part of $\boldsymbol{g}$. If $\bar{g}_{i, j}=1$, then there is a non-directed link between player $i$ and player $j$; this non-directed link is denoted by $[i j]$. A path of length $m$ in $\boldsymbol{g} \in \mathcal{G}$ from player $i$ to player $j \neq i$, is a finite sequence $i_{0}, i_{1}, \ldots, i_{m}$ of distinct players such that $i_{0}=i$, $i_{m}=j$, and $g_{i_{k}, i_{k+1}}=1$ for $k=0, \ldots, m-1$. We define a chain of length $m$ between player $k$ and player $j, j \neq k$ by replacing $\boldsymbol{g}_{j_{\ell}, j_{\ell+1}}=1$ by $\overline{\boldsymbol{g}}_{j_{\ell}, j_{\ell+1}}=1$. Let $\mathcal{C}_{i, j}(\boldsymbol{g})$ be the set of chains between $i$ and $j$ in the network $\boldsymbol{g}$, and let $C_{i, j}(\boldsymbol{g})$ be a typical element of $\mathcal{C}_{i, j}(\boldsymbol{g})$. To simplify we say that the non-directed link $\left[\ell \ell^{\prime}\right]$ belongs to a chain $C_{i, j}(\boldsymbol{g})$ if players $\ell$ and $\ell^{\prime}$ belong to the chain and $g_{\ell, \ell^{\prime}}=1$. A network $\boldsymbol{g}$ is connected if there is a chain in $\boldsymbol{g}$ between all players $i, j \in N$. Given a network $\boldsymbol{g}$, we define a component, $D(\boldsymbol{g})$, as a set of players such that there is a chain between any two players who belong to $D(\boldsymbol{g})$, and there does not exist a chain between a player in $D(\boldsymbol{g})$ and a player who does not belong to $D(\boldsymbol{g})$. We do not consider isolated players (players who do not form and receive any links) as components. A network $\boldsymbol{g}$ is connected if it contains only one component which consists of all players. It is minimally connected if it is not possible to preserve connectivity whenever a link 
is removed. A network is minimal if every component is minimally connected. A network $\boldsymbol{g}$ is called a star if there is a vertex $i_{s}$, such that for all $j \neq i_{s}, \bar{g}_{i_{s}, j}=1$ and for all $k \notin\left\{i_{s}, j\right\}, \bar{g}_{k, j}=0$. Moreover a star, where $g_{i_{s}, j}=1$ for all $j \neq i_{s}$ is a center-sponsored star. Finally, a network $\boldsymbol{g} \in \mathcal{G}$ is essential if $g_{i, j}=1$ implies $g_{j, i}=0$.

Payoffs. Payoffs of player $i$ are given by the difference between benefits $B_{i}(\boldsymbol{g})$ and $\operatorname{costs} c_{i}(\boldsymbol{g})$. Hence the payoff of player $i$ in network $\boldsymbol{g}$ is given by

$$
u_{i}(\boldsymbol{g})=B_{i}(\boldsymbol{g})-c_{i}(\boldsymbol{g}) .
$$

Next we define two types of heterogeneity in networks by introducing costs and different benefit formulations.

(i) Link Costs. Players incur costs only for the links they establish. The cost of each link is assumed to be the same, and the cost of forming links for player $i$ is given by:

$$
c_{i}(\boldsymbol{g})=\sum_{j \neq i} g_{i, j} c,
$$

with $c>0$. In this paper we only use homogeneous costs. Note that in our context heterogeneous costs would not improve anything because (i) they would only increase the set of potential strict Nash networks, and (ii) they would weaken the possibility of existence of Nash networks. This issue is discussed in detail in subsequent sections of the paper (see also Galeotti et al., 2006 for more on this).

(ii) Link Benefits. In the Nash networks setting, decay models were analyzed by Bala and Goyal (2000) who assumed that the value of information, the costs of link formation, and the decay parameter were identical across all players and links. In other words, they analyzed the case of homogeneous decay. Here we propose two different frameworks to study the interaction between heterogeneity and decay.

We denote by $N_{i}(\boldsymbol{g})=\{i\} \cup\{j \in N \backslash\{i\} \mid$ there exists a chain in $\boldsymbol{g}$ between $i$ and $j\}$, the set of players that player $i$ can access or "observe" in network $\boldsymbol{g}$. Since player 
$i$ can obtain resources from a player $j$ with whom she is linked due to the chain, it follows that there is two-way flow of information.

Decay with Heterogeneous Players. Here we use the homogeneous decay assumption in conjunction with the heterogeneous players framework of Galeotti, Goyal and Kamphorst (2006). Information received from $j$ is worth $V_{i, j} \geq 0$ to player $i$. Let $V^{M}=\max _{(i, j) \in N \times N}\left\{V_{i, j}\right\}$ and $V^{m}=\min _{(i, j) \in N \times N}\left\{V_{i, j}\right\}$. The benefits function can be written as:

$$
B_{i}(\boldsymbol{g})=\sum_{j \in N_{i}(\boldsymbol{g})} \delta^{d_{i, j}(\boldsymbol{g})} V_{i, j}
$$

where $\delta$ is the decay parameter and $d_{i, j}(\boldsymbol{g})$ is the geodesic distance in the shortest chain between $i$ and $j$ in $\boldsymbol{g}$. We set $d_{i, j}(\boldsymbol{g})=\infty$ if there is no chain between $i$ and $j$ in $g$.

Decay with Heterogeneous Links. In this model we capture the fact that the quantity of information a link can convey is not the same across all links under decay. In other words, some channels of information or chains are "better" than others.

We measure decay associated with a link $i j$ by the parameter $\delta_{i, j} \in[0,1]$. For this model we retain the symmetry assumption, that is $\delta_{i, j}=\delta_{j, i}$. Without loss of generality we assume that the value of the resources of each player $V=1$. Given a network $\boldsymbol{g}$, it is assumed that if player $i$ has formed a link with player $j$, then she receives information of value $\delta_{i, j}$ from $j$. The benefits of player $i$ in the network $\boldsymbol{g}$ is then given by:

$$
B_{i}(\boldsymbol{g})=\sum_{j \in N_{i}(\boldsymbol{g})}\left(\prod_{[\ell} \prod_{k] \in C_{i, j}^{*}(\boldsymbol{g})} \delta_{\ell, k}\right),
$$

where $C_{i, j}^{*}(\boldsymbol{g})=\arg \max _{C_{i, j}(\boldsymbol{g}) \in \mathcal{C}_{i, j}(\boldsymbol{g})}\left\{\prod_{[\ell k] \in C_{i, j}(\boldsymbol{g})} \delta_{\ell, k}\right\}$.

Note that this expression fundamentally differs from the previous one because it does not use the geodesic distance between players to determine the value of in- 
formation obtained.

Observe that in the model with heterogeneous decay, the amount of information that passes through a link between any two players depends on the identity of the players. On the other hand, with heterogeneous values the links between all players have the ability to convey the same amount of information, but the value that is acquired through a link now depends on the identity of the players.

Nash Networks. Given a network $\boldsymbol{g} \in \mathcal{G}$, let $\boldsymbol{g}_{-i}$ denote the network that remains when all of player $i$ 's links have been removed. Clearly, $\boldsymbol{g}=\boldsymbol{g}_{i} \oplus \boldsymbol{g}_{-i}$, where the symbol $\oplus$ indicates that $\boldsymbol{g}$ is composed of the union of links in $\boldsymbol{g}_{i}$ and $\boldsymbol{g}_{-i}$ (similarly the symbol $\ominus$ is used to indicate removal of links). A strategy $\boldsymbol{g}_{i}$ is a best response of player $i$ to $\boldsymbol{g}_{-i}$ if

$$
u_{i}\left(\boldsymbol{g}_{i} \oplus \boldsymbol{g}_{-i}\right) \geq u_{i}\left(\boldsymbol{g}_{i}^{\prime} \oplus \boldsymbol{g}_{-i}\right), \text { for all } \boldsymbol{g}_{i}^{\prime} \in \mathcal{G}_{i} .
$$

Let $\mathcal{B R}_{i}\left(\boldsymbol{g}_{-i}\right)$ denote the set of player $i$ 's best responses to $\boldsymbol{g}_{-i}$. A network $\boldsymbol{g}=$ $\left(\boldsymbol{g}_{1}, \ldots, \boldsymbol{g}_{n}\right)$ is said to be a Nash network if $\boldsymbol{g}_{i} \in \mathcal{B R}_{i}\left(\boldsymbol{g}_{-i}\right)$ for each $i \in N$. We define a strict best response and a strict Nash network by replacing " $\geq$ " with ">". Note that if $\boldsymbol{g} \in \mathcal{G}$ is a Nash network, then it must be essential. This follows from the fact that each link is costly, and allows for two-way flow of information regardless of who initiates (and pays for) the link.

\section{$3 \quad$ Results}

In this section we investigate Nash networks in the connections model with heterogeneity.

\subsection{Decay with Heterogeneous Players}

In this section we first examine the existence of Nash networks, then we characterize strict Nash networks. 
I. Existence of Nash networks. Existence of Nash networks in two-way flow models with decay has not been studied before in the literature. We know that the introduction of full heterogeneity on the costs of setting links without any decay can lead to non existence of Nash networks (see Haller, Kamphorst and Sarangi, 2007). Hence, we introduce heterogeneity in stages. We begin by showing that if heterogeneity is not "too high", more precisely if $V_{i, j}=V_{i}$ for all $i \in N$, then a Nash network always exists. It follows that there always exists a Nash network in the model of Bala and Goyal (2000).

Proposition 1 If the benefits function satisfies equation (3) and, for all $i \in N$, $V_{i, j}=V_{i}$, for all $j \in N \backslash\{i\}$, then a Nash network always exists.

Proof. Let $\mathcal{Z}_{0}=\left\{j \in N \mid \delta V_{j} \geq c\right\}$ be the set of players who have an incentive to form a link with any player $j$ with whom they are not (indirectly) linked; and let $z$ be the maximal value player, that is the player such that $V_{z} \geq V_{i}$ for all $i \in N$. Moreover, let $\mathcal{Z}_{1}=\left\{j \in N \mid\left(\delta-\delta^{2}\right) V_{j} \geq c\right\}$ be the set of players who have an incentive to form a link with any player $j$ with whom they are not directly linked. Clearly if $\mathcal{Z}_{1} \neq \emptyset$, then $z \in \mathcal{Z}_{1}$. Further if $\mathcal{Z}_{1}=\emptyset$, then no player $i$ will form a link with a player $j$ in $\boldsymbol{g}$ whenever $d_{i, j}(\boldsymbol{g}) \leq 2$. If $\mathcal{Z}_{0}=\emptyset$, then the empty network is a Nash network. If $\mathcal{Z}_{0} \neq \emptyset$ and $\mathcal{Z}_{1}=\emptyset$, then we let player $z$ form links with all other players. We obtain a center-sponsored star, $\boldsymbol{g}^{c s}$, which is a Nash network. Indeed, the distance between all players $i \in N \backslash\{z\}$ and $j \in N \backslash\{z, i\}$ is 2 in $\boldsymbol{g}^{c s}$ and we know that $\mathcal{Z}_{1}=\emptyset$. It follows that no player $i \in N \backslash\{z\}$ has an incentive to form a link with $j \in N \backslash\{z, i\}$ in $\boldsymbol{g}^{c s}$. If $\mathcal{Z}_{0} \neq \emptyset$ and $\mathcal{Z}_{1} \neq \emptyset$, then we create network $\boldsymbol{g}$ where player $z$ forms links with all other players and where $g_{j, i}=0$ implies $g_{i, j}=1$ for all $i \in \mathcal{Z}_{1}$ and for all $j \in N \backslash\{z\}$. Clearly, $\boldsymbol{g}$ is Nash since player $z \in \mathcal{Z}_{1}$ has no incentive to delete one of her links, each player $i \in \mathcal{Z}_{1}$ has no incentive to delete any link by construction and no player $i^{\prime} \notin \mathcal{Z}_{1}$ has any incentive to form an additional link (otherwise she would belong to $\mathcal{Z}_{1}$ ). It follows that a Nash network always exists. 
Although Bala and Goyal (2000) are able to provide a partial characterization of strict Nash networks in the model with decay and homogeneous parameters model, they do not tackle the existence question. Existence of strict Nash networks in the homogeneous parameters model with decay is now an obvious corollary of Proposition 1.

Corollary 1 Let the payoff function be the one given in Bala and Goyal (2000, pg.1210). Then a Nash network always exists.

Proposition 2 If the benefits function satisfies equation (3), then a Nash network does not always exist.

Proof. The proof is given through an example. Let $N=\{1, \ldots, 5\}$ be the set of players, and assume that:

1. $V_{1,2}\left(\delta-\delta^{4}\right)+V_{1,3}\left(\delta^{2}-\delta^{3}\right)>c, \delta V_{1,3}<\delta V_{1,2}<c$, and for all $j \neq 2, \delta V_{1, j}+$ $\delta^{2} \sum_{k \neq j} V_{1, k}<c$.

2. $V_{2,3}\left(\delta-\delta^{4}\right)+V_{2,4}\left(\delta^{2}-\delta^{3}\right)<c, \delta V_{2,3}+\delta^{2} V_{2,4}+\delta^{3} V_{2,5}+\delta^{4} V_{2,1}>c$, and for all $j \neq 3, \delta V_{2, j}+\delta^{2} \sum_{k \neq j} V_{2, k}<c$.

3. $\left(\delta-\delta^{2}\right) V_{3,4}>c$ and $\delta \sum_{k \neq 4} V_{3, k}+\delta^{2} V_{3,4}<c$.

4. $\left(\delta-\delta^{2}\right) V_{4,5}>c$ and $\delta \sum_{k \neq 5} V_{4, k}+\delta^{2} V_{4,5}<c$.

5. $\left(\delta-\delta^{2}\right) V_{5,1}>c$ and $\delta \sum_{k \neq 1} V_{5, k}+\delta^{2} V_{5,1}<c$.

These five points provide a list of the players with whom the others have no incentives to form links, as well as those with whom they would like to form links. For example, item 1 implies that player 1 will never form a link with players 3,4 and 5 . Moreover, a Nash network must contain the links $34,45,51$. From all of this, it follows that there are four possible Nash networks: $E\left(\boldsymbol{g}^{1}\right)=(34,45,51,12,23)$, $E\left(\boldsymbol{g}^{2}\right)=(34,45,51,12), E\left(\boldsymbol{g}^{3}\right)=(34,45,51), E\left(\boldsymbol{g}^{4}\right)=(34,45,51,23)$. We know from item 2 that player 2 prefers the network $\boldsymbol{g}^{2}$ to the network $\boldsymbol{g}^{1}$, so $\boldsymbol{g}^{1}$ is not Nash. Likewise, player 1 prefers the network $\boldsymbol{g}^{3}$ to the network $\boldsymbol{g}^{2}$ by point 1 , 
so $\boldsymbol{g}^{2}$ is not Nash. Player 2 prefers the network $\boldsymbol{g}^{4}$ to the network $\boldsymbol{g}^{3}$ by point 2, so $\boldsymbol{g}^{3}$ is not Nash. Finally, by point 1, player 1 prefers the network $\boldsymbol{g}^{1}$ to the network $\boldsymbol{g}^{4}$. Hence $\boldsymbol{g}^{4}$ is not Nash.

This result provides an interesting comparison with the findings of Haller, Kamphorst and Sarangi (2007). The authors show that in the model with no decay, value heterogeneity (and homogeneous cost) does not lead to non existence (2007, Proposition, pg.602) while cost heterogeneity does (2007, Example 2, pg.602). By contrast however in a model with homogeneous costs and decay, Proposition 2 shows that value heterogeneity can lead to non-existence.

II. Characterization of Strict Nash networks. We begin by showing that the introduction of player heterogeneity dramatically increases the set of strict Nash networks.

Theorem 1 Let $\boldsymbol{g}$ be an essential network. If the benefits function satisfies equation (3), then there exist a link cost $c>0$, a decay $\delta \in(0,1)$, and an array $\boldsymbol{V}=\left[V_{i, j}\right]$ of values such that $\boldsymbol{g}$ is a strict Nash network in the corresponding network formation game.

Proof. Suppose $\boldsymbol{g}$ is an essential network. Let $V^{1}=1, c=(n-3 / 2) / n^{2}, \delta=1 / n$, $V^{0}=1 /(3 n)$. We construct a symmetric $n \times n$-matrix $\left[V_{i, j}\right]$ of value as follows. If $i \neq j$ and $i$ and $j$ are linked, i.e. $g_{i, j}=1$ or $g_{j, i}=1$ set $V_{i, j}=V^{1}$. Otherwise set $V_{i, j}=V^{0}$. Now consider $i \neq j$. Let $g_{i, j}=0$. Then, either $g_{j, i}=1$ or $g_{j, i}=0$. In the first case, agent $i$ receives zero marginal benefits but incurs an additional positive cost when forming the link $i j$. It follows that $g_{i, j}=0$ is the unique optimal choice for $i$ given $\boldsymbol{g}_{-i}$. For $g_{j, i}=0, V_{i, j}=V^{0}=1 /(3 n)$. If player $i$ forms a link with $j$, then she obtains at most marginal benefits equal to $\delta V^{0}+(n-2) \delta^{2} V^{1}$. We show that $\delta V^{0}<c-(n-2) \delta^{2} V^{1}$. We have $\delta V^{0}=$ $1 /\left(3 n^{2}\right)<1 /\left(2 n^{2}\right)=(n-3 / 2) / n^{2}-(n-2) / n^{2}=c-(n-2) \delta^{2} V^{1}$. Therefore regardless of other links, not initiating the link $i j$ is optimal for agent $i$. Now let 
$g_{i, j}=1$. Then by essentiality of $\boldsymbol{g}, g_{j, i}=0$. Further, $V_{i, j}=V^{1}$. If player $i$ removes the link $i j$, then she obtains at most a payoff equal to $\delta^{2} V_{i, j}$ from player $j$. It follows that due to the link $i j$ player $i$ obtains marginal benefits equal to at least $V^{1}\left(\delta-\delta^{2}\right)=1 / n-1 / n^{2}=(n-1) / n^{2}>(n-(3 / 2)) / n^{2}=c$. Therefore regardless of other links, player $i$ has no incentive to remove the link $i j$.

From the above proposition we can establish two things. First, consider the following quote from Galeotti et al. (2006, pg. 360): “This shows that, in case of general cost heterogeneity, the level of value heterogeneity plays no important role in determining the network architecture." This observation is not true for models with decay where regardless of the level of cost heterogeneity, value heterogeneity is important for determining the network architecture.

The second point is about how the role value heterogeneity in a model of partial transitivity compares to the role cost heterogeneity in a model with full transitivity. We know that value heterogeneity with homogeneous cost and no decay leads to strict Nash networks which are either the empty network, or minimal networks in which every (non-singleton) component is a center-sponsored star (see Galeotti et al., 2006, Proposition 3.1, pg.359). We also know that under cost heterogeneity and homogeneous value every minimal network can be supported as a strict Nash equilibrium in the absence of decay (see Galeotti et al., 2006, Proposition 3.2, pg.360). Since there is no decay, this model allows for full transitivity in information acquisition. Consequently, every strict Nash network must be minimal regardless of parameter value. Thus Proposition 3.2 of Galeotti et al. identifies the largest permissible set of networks as strict Nash networks. The introduction of (homogeneous) decay however leads to partial transitivity in the model. In this situation, we find that value heterogeneity alone has the potential to make every network strict Nash, which coincides with the largest permissible set of networks. To borrow from Galeotti et al., value heterogeneity "... is important in shaping both the level of connectedness as well as the architecture of individual components." Thus, in a model of decay, value heterogeneity plays the same role as cost heterogeneity in a model with full transitivity. 
With homogeneous players, strict Nash networks are either empty or connected (see Bala and Goyal, 2000). A natural question that arises in the context of the heterogeneous players model is when can such a result be found for this model? We show next that with heterogeneous values it is possible to obtain this result if the values of players are sufficiently close.

Proposition 3 Suppose benefits function satisfies equation (3) and that $V^{M}-$ $V^{m}<\delta V^{m} /(1+(n-3) \delta)$. Then a strict Nash network is either empty or connected.

Proof. Let $D^{*}(\boldsymbol{g}, i j)$ be the set of players $\ell \in N \backslash\{i, j\}$ such that the shortest chain between $i$ and $\ell$ goes through the link $i j$ in $\boldsymbol{g}$. Consider a strict Nash network $\boldsymbol{g}$. Suppose $\boldsymbol{g}$ is neither empty nor connected. Then there exist three agents $i, j$ and $k$ such that $i$ and $j$ belong to one connected component $D_{1}$ and $k$ belongs to a different component $D_{2}$ in $\boldsymbol{g}$. Moreover, wlog let $g_{i, j}=1$. Then the incremental benefits to player $i$ of having the direct link to $j$ is given by:

$$
\begin{aligned}
\Delta^{M} & =\left(\delta-\delta^{d_{i, j}(\boldsymbol{g} \ominus i j)}\right) V_{i, j}+\sum_{\ell \in D^{*}(\boldsymbol{g}, i j)}\left(\delta^{d_{i, \ell}(\boldsymbol{g})}-\delta^{d_{i, \ell}(\boldsymbol{g} \ominus i j)}\right) V_{i, \ell} \\
& \leq \delta V_{i, j}+\sum_{\ell \in D^{*}(\boldsymbol{g}, i j)} \delta^{d_{i, \ell}(\boldsymbol{g})} V_{i, \ell} \\
& \leq \delta V^{M}+\sum_{\ell \in D^{*}(\boldsymbol{g}, i j)} \delta^{d_{i, \ell}(\boldsymbol{g})} V^{M}
\end{aligned}
$$

with the convention $\delta^{d_{i, \ell}(\boldsymbol{g} \ominus i j)}=0$, if $\ell \notin N_{i}(\boldsymbol{g} \ominus i j)$. Clearly, we have $\Delta^{M} \geq c$.

If player $k$ forms a link with player $j$, then the incremental benefits to player $i$ of having the direct link to $j$ is:

$$
\begin{aligned}
\Delta^{m} & \geq \delta V_{k, j}+\delta^{2} V_{k, i}+\sum_{\ell \in D^{*}(\boldsymbol{g}, i j)} \delta^{d_{i, \ell}(\boldsymbol{g})} V_{k, \ell} \\
& \geq \delta V^{m}+\delta^{2} V^{m}+\sum_{\ell \in D^{*}(\boldsymbol{g}, i j)} \delta^{d_{i, \ell}(\boldsymbol{g})} V^{m} .
\end{aligned}
$$

It is worth noting that:

$$
\sum_{\ell \in D^{*}(\boldsymbol{g}, i j)} \delta^{d_{i, \ell}(\boldsymbol{g})} V^{M}-\sum_{\ell \in D^{*}(\boldsymbol{g}, i j)} \delta^{d_{i, \ell}(\boldsymbol{g})} V^{m} \leq(n-3) \delta^{2}\left(V^{M}-V^{m}\right) .
$$


Since $V^{M}-V^{m}<\delta V^{m} /(1+(n-3) \delta)$, we have $\Delta^{m}>\Delta^{M} \geq c$. It follows that player $k$ has a incentive to form a link with $j$ and $\boldsymbol{g}$ is not strict Nash.

Not surprisingly Proposition 3 also tells us that in the homogeneous parameters model with decay not every network can be supported as a strict Nash network. Indeed, it is easy to see that $V^{M}-V^{m}<\delta V^{m} /(1+(n-3) \delta)$ is always satisfied in the homogeneous model. Therefore, non-connected networks cannot be strict Nash.

In the above proposition we restrict heterogeneity by putting a bound on its magnitude. Now we restrict the heterogeneity by assuming that $V_{i, j}=V_{i}$. Recall that reducing heterogeneity in this manner has positive implications for the existence of Nash networks. Below we show how it affects the set of strict Nash networks.

Proposition 4 Suppose payoff function satisfies equation (3) and for each player $i \in N, V_{i, j}=V_{i}$ for all $j \in N$. Then a strict Nash network contains at most one component.

Proof. Suppose $\boldsymbol{g}$ is a strict Nash network and contains two components $D$ and $D^{\prime}$. Let $i, j \in D$ and $i^{\prime}, j^{\prime} \in D^{\prime}$ be such that $g_{i, j}=g_{i^{\prime}, j^{\prime}}=1$ and $V_{i} \geq V_{i^{\prime}}$. Let $\boldsymbol{g}^{0}=\boldsymbol{g} \ominus i^{\prime} j^{\prime}, \mathcal{A}=\left\{\ell \in N_{i^{\prime}}(\boldsymbol{g}) \mid d^{i^{\prime}, \ell}(\boldsymbol{g})<d^{i^{\prime}, \ell}\left(\boldsymbol{g}^{0}\right)\right\}$ and $\mathcal{B}=\left\{\ell \in N_{i^{\prime}}(\boldsymbol{g}) \mid d^{i^{\prime}, \ell}\left(\boldsymbol{g}_{-i^{\prime}} \oplus\right.\right.$ $\left.\left.i^{\prime} j^{\prime}\right)=d^{i^{\prime}, \ell}\left(\boldsymbol{g}^{0}\right)\right\}$. We have:

$$
\Delta_{i^{\prime}}=u_{i^{\prime}}(\boldsymbol{g})-u_{i^{\prime}}\left(\boldsymbol{g}^{0}\right)=\sum_{\ell \in \mathcal{A}}\left(\delta^{d^{i^{\prime}, \ell}(\boldsymbol{g})}-\delta^{d^{i^{\prime}, \ell}\left(\boldsymbol{g}^{0}\right)}\right) V_{i^{\prime}}-c
$$

with the convention $\delta^{d_{i^{\prime}, \ell}\left(\boldsymbol{g}^{0}\right)}=0$, if $\ell \notin N_{i}\left(\boldsymbol{g}^{0}\right)$. Since $\boldsymbol{g}$ is strict Nash, we have $\Delta_{i^{\prime}}>0$.

Let $\boldsymbol{g}^{1}=\boldsymbol{g} \oplus i j^{\prime}$, we have:

$$
\begin{aligned}
\Delta_{i}=u_{i}\left(\boldsymbol{g}^{1}\right)-u_{i}(\boldsymbol{g})= & \sum_{\ell \in \mathcal{A}} \delta^{d^{i^{\prime}, \ell}(\boldsymbol{g})} V_{i}+\sum_{\ell \in \mathcal{B} \cup\left\{i^{\prime}\right\}} \delta^{d^{i^{\prime}, \ell}(\boldsymbol{g})} V_{i} \\
& +\sum_{\ell \in N_{i^{\prime}}(\boldsymbol{g}) \backslash(\mathcal{A} \cup \mathcal{B})}\left(\delta^{d^{i^{\prime}, \ell}(\boldsymbol{g})+1}\right) V_{i}-c
\end{aligned}
$$

Since $\boldsymbol{g}$ is strict Nash, we have $\Delta_{i}<0$. We obtain a contradiction since $\Delta_{i^{\prime}} \leq \Delta_{i}$. 
Proposition 4 shows that when we reduce the heterogeneity by restricting player $i$ to acquire $V_{i}$ from each of the players she observes, not every essential network can be strict Nash. This sharply contrasts with the heterogeneous players case of Theorem 1. Moreover, the model where values are given by $V_{i}$ allows for strict Nash networks that never arise in the homogeneous parameters model. Indeed, we know by Proposition 3 that with low value heterogeneity (with the homogeneous decay model being the limiting case) non-empty strict Nash networks contain one component, say $D$, and all players belong to $D$. In the following example, we show that when values are given by $V_{i}$, in a strict Nash network isolated players can also co-exist with a single component.

Example 1 Consider $N=\{1, \ldots, 5\}, V_{i}=1$ for all $i \in N \backslash\{5\}$ and $V_{5}=\varepsilon$. We suppose that $4 \varepsilon<\delta<c$, and $\delta+2 \delta^{2}>c$. Then the network $\boldsymbol{g}$ drawn in Figure 1 is a strict Nash network.

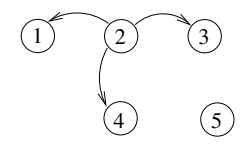

Figure 1: Network $\boldsymbol{g}$

\subsection{Decay with Heterogeneous Links}

In this section we consider situations where players have homogeneous values while the decay through each link is different.

\section{Existence of Nash networks.}

Proposition 5 Suppose the benefits function satisfies equation (4), then a Nash network does not always exist.

Proof. The proof is given through a counter-example. Let $N=\{1,2,21,3,31, \ldots$, $36,4,41\}$ be the set of players. We assume that $c=0.95, \delta_{1,2}=\delta_{2,1}=0.4$, 
$\delta_{1,4}=\delta_{4,1}=0.319, \delta_{4,3}=\delta_{3,4}=0.14, \delta_{2,3}=\delta_{3,2}=0.1303, \delta_{2,21}=\delta_{21,2}=1$, $\delta_{4,41}=\delta_{41,4}=1, \delta_{3, k}=\delta_{k, 3}=1$, for all $k \in\{31, \ldots, 36\}$, and $\delta_{i, j}=0$ for all remaining $(i, j) \in N \times N \backslash\{i\}$. Obviously, none of the links with $\delta_{i, j}=0$ will be established, and all links $i j$ such that $\delta_{i, j}=1$ will be established. From this observation we know that in a Nash network $\boldsymbol{g}$ we have $\bar{g}_{2,21}=\bar{g}_{3,31}=\ldots=\bar{g}_{3,36}=\bar{g}_{4,41}=1$. For candidate Nash networks, we consider the set $A$ containing the following links: $\{221,331, \ldots, 336,441\}$. Although links $212,313, \ldots$ are possible for the arguments used below, the non existence result is not affected by them. Therefore without loss of generality we focus on the set $A$. Moreover, straightforward computations allow us to conclude that player 3 forms no links except with players $31, \ldots, 36$, players 2 and 4 cannot form a link with player 1 . Similarly, it can be checked that player 1 will not form a link with player 3. Likewise, player 2 and player 4 will not form links with each other. In addition to the set of links in $A$, it follows that the links that can be formed by players in a Nash network also belong to the set $B=\{12,14,23,43\}$. Therefore, the set of potential Nash networks consists of networks for which the edge set is a subset of $A \cup B$. In Figure 2 each box contains the links associated with $\delta=1$. The other links are associated with the non-zero values of $\delta$.

1. Let $\boldsymbol{g}^{0}$ be a network such that $E\left(\boldsymbol{g}^{0}\right)=A$. Then $\boldsymbol{g}^{0}$ is not Nash since player 4 has an incentive to form a link with player 3 .

2. We are now interested in networks which have $|A|+1$ links. Straightforward computations show that only $\boldsymbol{g}^{1}$, with $E\left(\boldsymbol{g}^{1}\right)=A \cup\{43\}$ is candidate to be Nash. Indeed, in other networks with $|A|+1$ links, the player who forms the additional link has no incentive to maintain it. Network $\boldsymbol{g}^{1}$ is not Nash since player 1 has an incentive to form a link with player 4 .

3. We are now interested in networks which have $|A|+2$ links. Straightforward computations show that only $\boldsymbol{g}^{2}$, with $E\left(\boldsymbol{g}^{2}\right)=A \cup\{14,43\}$ is candidate to be Nash. This network is not Nash since player 2 has an incentive to form a link with player 3 . 
4. We are now interested in networks which have $|A|+3$ links. Straightforward computations show that only $\boldsymbol{g}^{3}$, with $E\left(\boldsymbol{g}^{3}\right)=A \cup\{14,23,43\}$ and $\boldsymbol{g}^{4}$, with $E\left(\boldsymbol{g}^{4}\right)=A \cup\{12,23,43\}$ are candidates to be Nash. Network $\boldsymbol{g}^{3}$ is not Nash since player 1 prefers network $\boldsymbol{g}^{4}$. Network $\boldsymbol{g}^{4}$ is not Nash since player 2 has an incentive to delete the link 23 .

5. We are now interested in the network with $|A|+4$ links which is a candidate to be a Nash network. In this network, player 1 has an incentive to remove the link 14 .

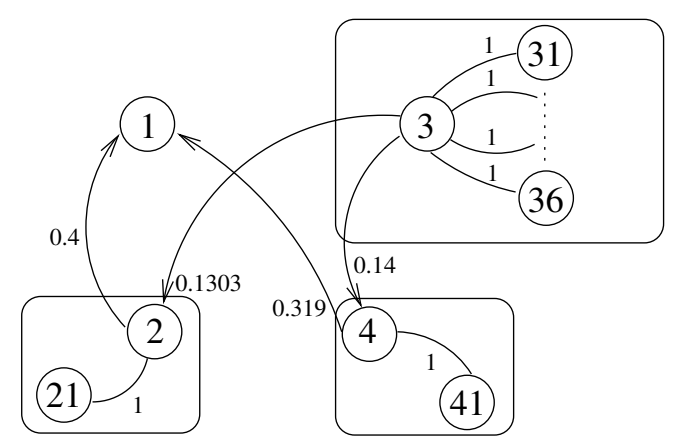

Figure 2:

Note that when we set the decay parameter $\delta_{i, j}=\delta_{i}$ it is easy to show that a Nash network always exists.

II. Characterization of Strict Nash Networks. Results regarding strict Nash networks in this model are similar to what we find in the previous model. The exact analogue of Theorem 1 can be written for this model. Similarly, we can reduce the magnitude of the decay parameter and find a result equivalent to Proposition $3 .^{4}$

\footnotetext{
${ }^{4}$ Details can be found in the working paper version: http: \|bus.lsu.edu $\backslash$ mcmillin $\backslash$ Working_Papers $\backslash$ pap10_04.pdf
} 


\section{Concluding Remarks}

In this paper we investigate the implications of heterogeneity in the value parameter and decay parameter in the connections model. We find that both types of heterogeneity are similar to cost heterogeneity in models of full transitivity, i.e. they increase the size of strict Nash networks to the maximum possible and can lead to non-existence of Nash networks. Moreover, value heterogeneity in the connections model is different from value heterogeneity in the full transitivity model where it does not dramatically increase the size of strict Nash networks, nor does it lead to non-existence. Cost heterogeneity however has the same type of effect both in models of full and partial transitivity.

\section{References}

[1] V. Bala and S. Goyal. A Non-Cooperative Model of Network Formation. Econometrica, 68(5):1181-1229, 2000.

[2] A. Galeotti, S. Goyal, and J. Kamphorst. Network Formation with Heteregeneous Players. Games and Economic Behavior, 54(2):353-372, 2006.

[3] H. Haller, J. Kamphorst, and S. Sarangi. (Non-)Existence and Scope of Nash Networks. Economic Theory, 31(3):597-604, 2007.

[4] Daniel A. Hojman and Adam Szeidl. Core and periphery in networks. Journal of Economic Theory, 139(1):295-309, March 2008.

[5] M. Jackson and A. Wolinsky. A Strategic Model of Social and Economic Networks. Journal of Economic Theory, 71(1):355-365, 1996. 